TRANSACTIONS OF THE

AMERICAN MATHEMATICAL SOCIETY

Volume 349, Number 1, January 1997, Pages 287-310

S $0002-9947(97) 01652-8$

\title{
SUMS OF THREE OR MORE PRIMES
}

\author{
J. B. FRIEDLANDER AND D. A. GOLDSTON
}

\begin{abstract}
It has long been known that, under the assumption of the Riemann Hypothesis, one can give upper and lower bounds for the error $\sum_{p \leq x} \log p-x$ in the Prime Number Theorem, such bounds being within a factor of $(\log x)^{2}$ of each other and this fact being equivalent to the Riemann Hypothesis. In this paper we show that, provided "Riemann Hypothesis" is replaced by "Generalized Riemann Hypothesis", results of similar (often greater) precision hold in the case of the corresponding formula for the representation of an integer as the sum of $k$ primes for $k \geq 4$, and, in a mean square sense, for $k \geq 3$. We also sharpen, in most cases to best possible form, the original estimates of Hardy and Littlewood which were based on the assumption of a "Quasi-Riemann Hypothesis". We incidentally give a slight sharpening to a well-known exponential sum estimate of Vinogradov-Vaughan.
\end{abstract}

\section{INTRODUCTION}

Let

$$
r(n)=r_{k}(n)=\sum_{p_{1}+p_{2}+\cdots+p_{k}=n}\left(\log p_{1}\right)\left(\log p_{2}\right) \cdots\left(\log p_{k}\right)
$$

denote the weighted number of representations of $n$ as the sum of $k$ primes. For $k \geq 2$ the expected asymptotic formula for $r(n)$ is of the form

$$
r_{k}(n)=\frac{1}{(k-1) !} n^{k-1} \mathfrak{S}_{k}(n)+\text { error },
$$

where $\mathfrak{S}_{k}$ is the singular series

$$
\mathfrak{S}_{k}(n)=\sum_{q=1}^{\infty} \frac{\mu^{k}(q)}{\phi^{k}(q)} c_{q}(-n)=\prod_{p \mid n}\left(1-\left(\frac{-1}{p-1}\right)^{k-1}\right) \prod_{p \nmid n}\left(1-\left(\frac{-1}{p-1}\right)^{k}\right),
$$

$c_{q}(n)$ is the Ramanujan sum

$$
c_{q}(n)=\sum_{\substack{1 \leq a \leq q \\(a, q)=1}} e\left(\frac{a n}{q}\right),
$$

and $e(u)=e^{2 \pi i u}$. Thanks to Vinogradov [Vi], it is known that (1.1) holds in case $k \geq 3$. The best error with which it is known to hold is $O\left(n^{k-1}(\log n)^{-A}\right)$, for

Received by the editors April 29, 1994 and, in revised form, September 22, 1995.

1991 Mathematics Subject Classification. Primary 11P32.

Research of the first author supported in part by NSERC Grant A5123 and NSF Grant DMS8505550.

Research of the second author supported in part by NSF Grant DMS9205533.

(C)1997 American Mathematical Society 
arbitrary $A,\left(\mathrm{cf}[\mathrm{Da}],\left[\mathrm{Va}_{2}\right]\right)$. Note that, for $k \geq 3$, as long as the 2-adic factor in (1.2) does not vanish, we have $1 \ll \mathfrak{S}_{k}(n) \ll 1$.

In this paper we are interested in the conditions under which one may, for $k \geq 3$, improve the error term in (1.1), for example to obtain

$$
r_{k}(n)=\frac{1}{(k-1) !} n^{k-1} \mathfrak{S}_{k}(n)+O\left(n^{k-1-\delta+\epsilon}\right)
$$

for various $\delta$ and for all $\epsilon>0$. Indeed one knows [MV, p. 208] that, if we define $\Theta$ by

$$
\Theta=\sup \{\beta: L(\beta+i \gamma, \chi)=0\}
$$

where the supremum is over all zeros of all the $L$-functions for Dirichlet characters $\chi$, then the constant $\delta$ in $(1.3)$ cannot exceed $1-\Theta$. Thus, to unconditionally prove (1.3) with some positive $\delta$ for some $k$ would imply a quasi-Riemann hypothesis, namely that no Dirichlet $L$-function of any modulus can have a zero with real part exceeding $1-\delta$.

The first result of type (1.3) was established by Hardy and Littlewood in their pioneering paper [HL] which inspired the subsequent unconditional proof of (1.1) for $k \geq 3$ by Vinogradov. They proved, for $k \geq 3$ and some $B$,

$$
r_{k}(n)=\frac{1}{(k-1) !} n^{k-1} \mathfrak{S}_{k}(n)+O\left(n^{k-7 / 4+\Theta}(\log n)^{B}\right)
$$

which gives (1.3) with $\delta=1-\Theta-1 / 4$, and is thus non-trivial in case $\Theta<3 / 4$.

In our first theorem we show that, even for larger $\Theta$, one can get a result of this type, and moreover one can, in all but a few cases, replace the Hardy-Littlewood error term by one in which the exponent is best possible. By the above-mentioned result of $[\mathrm{MV}]$ we know that one cannot improve the exponent in the error term in

$$
r_{k}(n)=\frac{1}{(k-1) !} n^{k-1} \mathfrak{S}_{k}(n)+O_{\epsilon}\left(n^{k-2+\Theta+\epsilon}\right)
$$

apart from the epsilon. Here we establish this bound for each $k \geq 5$ and get a slightly weaker estimate for $k=3$ and 4 . To do this we make use of "zero-density" bounds for $N(\sigma, T, \chi)$, the number of zeros $\beta+i \gamma$ of $L(s, \chi)$ satisfying $\beta \geq \sigma$, $0 \leq \gamma \leq T$.

Theorem 1. Let $k \geq 3$, let $\Theta$ be given by (1.4), and let $\epsilon>0$.

(a) We have

$$
r_{k}(n)=\frac{1}{(k-1) !} n^{k-1} \mathfrak{S}_{k}(n)+O\left(n^{k-2+\Theta+\epsilon}\right)+O\left(n^{\eta_{k}+\epsilon}\right),
$$

where $\eta_{3}=9 / 5, \eta_{4}=13 / 5$, and $\eta_{k}=0$ for $k \geq 5$.

(b) The exponents $9 / 5,13 / 5$ in (a) can be replaced by $7 / 4,5 / 2$ provided that we assume the "Density Conjecture" that

$$
\sum_{\chi \bmod q} N(\sigma, T, \chi) \ll(q T)^{2(1-\sigma)+\epsilon}
$$

holds for $1 / 2 \leq \sigma \leq 1$.

Thus, specifically, we obtain the best possible exponent $\delta$, that is $\delta=1-\Theta$, in the following ranges: for $k=3, \Theta \geq 4 / 5$ in (a) and $\Theta \geq 3 / 4$ in (b), for $k=4$, $\Theta \geq 3 / 5$ in (a) and all $\Theta$ in (b), and finally, for $k \geq 5$ and all $\Theta$. 
The zero-density estimates give weak results for $\Theta$ near $1 / 2$ and Theorem 1 can be sharpened in that case for $k=3,4$. The epsilons in (1.6) can also be sharpened and, in case $k \geq 4$, the first one can be dropped entirely.

Our primary concern in this paper is, however, with the case $\Theta=1 / 2$, the Generalized Riemann Hypothesis (GRH). In this, the most important case, we are interested in narrowing as much as possible the gap between the upper and lower bounds for the error term. Our second result gives an upper bound in this case.

Theorem 2. Assume GRH. We have

$$
\begin{aligned}
& r_{3}(n)=\frac{1}{2} n^{2} \mathfrak{S}_{3}(n)+O\left(n^{7 / 4}(\log n)^{5 / 2}\right), \\
& r_{4}(n)=\frac{1}{3 !} n^{3} \mathfrak{S}_{4}(n)+O\left(n^{5 / 2}(\log n)^{4}\right),
\end{aligned}
$$

and, for $k \geq 5$,

$$
r_{k}(n)=\frac{1}{(k-1) !} n^{k-1} \mathfrak{S}_{k}(n)+O\left(n^{k-3 / 2}\right) .
$$

By the above remarked statements of $[\mathrm{MV}]$ this gives

Corollary. Fix an integer $k \geq 5$. The bound (1.10) holds if and only if the Generalized Riemann Hypothesis is true.

and also the corresponding, slightly weaker statement for $k=4$.

Montgomery and Vaughan [MV, Theorem 1] have given, for all $k \geq 2$,

$$
r_{k}(n)-\frac{1}{(k-1) !} n^{k-1} \mathfrak{S}_{k}(n)=\Omega_{-}\left(n^{k-3 / 2}\right) .
$$

Thus, on GRH, the upper and lower bounds for the error term in (1.1) are, for $k \geq 5$, the same apart from the implied constant, and for $k=4$, are within a modest power of the logarithm of each other. The results (1.9) and (1.10) sharpen statements in [MV, p. 208] in which the bounds contain a factor $n^{\epsilon}$, and (1.8) gives an explicit value $B=5 / 2$ in the result (1.5) of Hardy and Littlewood.

In the case $k=3$ the exponent $7 / 4$ in (1.8) can be lowered to the expected value $k-3 / 2=3 / 2$ by making an additional assumption, see $\S 3$. In this case we are unable to use GRH alone to obtain the expected exponent in the error term. In our next theorem we are however able to do this in a mean square sense.

Theorem 3. Assume GRH. We have

$$
\begin{aligned}
& \sum_{n \leq N}\left(r_{3}(n)-\frac{1}{2} n^{2} \mathfrak{S}_{3}(n)\right)^{2} \ll N^{4}(\log N)^{7}, \\
& \sum_{n \leq N}\left(r_{4}(n)-\frac{1}{6} n^{3} \mathfrak{S}_{4}(n)\right)^{2} \ll N^{6}(\log N)^{4},
\end{aligned}
$$

and, for $k \geq 5$,

$$
\sum_{n \leq N}\left(r_{k}(n)-\frac{1}{(k-1) !} n^{k-1} \mathfrak{S}_{k}(n)\right)^{2} \ll N^{2 k-2} .
$$


Remarks. Of course, for $k \geq 5$, Theorem 3 is already an immediate corollary of (1.10). It may be possible, using work of Yildirim [Yi], to remove some of the above logarithmic factors, subject to the additional assumption of a pair correlation conjecture for the zeros of $L(s, \chi)$.

Our next result, obtained by slightly modifying the arguments in [MV], contains (1.11) apart from the sign, and serves as a complement to Theorem 3 in the same fashion that (1.11) does for Theorem 2.

Theorem 4. Let $k \geq 2$. The mean square on the left hand side in Theorem 3 is $\Omega\left(N^{2 k-2}\right)$.

Thus, in this case, even for $k=3$, it is only a power of the logarithm that is at issue between the upper and lower bounds. Note that, in the case $k=2,[\mathrm{MV}]$ gives Theorem 4 in the sharper form $\Omega\left(N^{2}(\log N)^{2}\right)$. A similar sharpening cannot, by Theorem 3 , occur for $k \geq 5$.

In the course of proving Theorem 1 we are led to a familiar exponential sum which we bound using estimates for the density of zeros of $L$-functions. The use of this technique in this context dates to Linnik [Li] and was later employed again by Montgomery $[\mathrm{Mo}]$ in conjunction with his much sharper density theorems. Let $(a, q)=1, \alpha=a / q+\beta$,

$$
S(\alpha)=\sum_{p \leq N}(\log p) e(p \alpha)
$$

and

$$
S_{1}(\beta ; q, a)=S\left(\frac{a}{q}+\beta\right)-\frac{\mu(q)}{\phi(q)} I(\beta),
$$

where $I(\beta)=\sum_{n \leq N} e(n \beta)$.

We first recall some well-known results. The following GRH estimate for $S_{1}$ is essentially due to Hardy-Littlewood, (but refined by Baker-Harman [BH], Goldston [Go]): Assume GRH. Then

$$
S_{1}(\beta ; q, a) \ll q^{1 / 2} N^{1 / 2} \log N\left(\log N+|\beta|^{1 / 2} N^{1 / 2}\right) .
$$

Vaughan $\left[\mathrm{Va}_{1}\right]$ sharpened and greatly simplified the proof of the unconditional estimate of Vinogradov giving: Let $|\beta| \leq 1 / q^{2}$. Then

$$
S_{1}(\beta ; q, a) \ll\left(q^{1 / 2} N^{1 / 2}+N^{4 / 5}+q^{-1 / 2} N\right)(\log N)^{4} .
$$

To these we add the following estimates.

Theorem 5. Let $\epsilon>0,|\beta| \leq 1 / q^{2}, T_{0}=1+|\beta| N$.

(a) We have

$$
S_{1}(\beta ; q, a) \ll_{\epsilon}\left\{\left(q T_{0}\right)^{1 / 2} N^{1 / 2}+\left(q T_{0}\right)^{1 / 4} N^{11 / 16}+q^{-1 / 2} N\right\} N^{\epsilon},
$$

and

$$
S_{1}(\beta ; q, a) \ll_{\epsilon}\left\{\left(q T_{0}\right)^{1 / 2} N^{1 / 2}+\left(q T_{0}\right)^{7 / 22} N^{7 / 11}+\left(q T_{0}\right)^{1 / 10} N^{3 / 4}+q^{-1 / 2} N\right\} N^{\epsilon} .
$$

(b) Let, in addition, $\Theta \leq \theta \leq 1$, where $\Theta$ is given in (1.4). Then

$$
\begin{aligned}
S_{1}(\beta ; q, a) \ll_{\epsilon}\left\{\left(q T_{0}\right)^{1 / 2} N^{1 / 2}+\right. & \left(q T_{0}\right)^{7 / 22} N^{7 / 11}+\left(q T_{0}\right)^{1 / 10} N^{3 / 4} \\
& \left.+q^{-1 / 50} N^{4 / 5}+q^{-2(\theta-3 / 4)} N^{\theta}\right\} N^{\epsilon},
\end{aligned}
$$


and, if one assumes that the density conjecture (1.7) holds for $\sigma \geq 1 / 2$, then

$$
S_{1}(\beta ; q, a) \ll_{\epsilon}\left\{\left(q T_{0}\right)^{1 / 2} N^{1 / 2}+q^{-2(\theta-3 / 4)} N^{\theta}\right\} N^{\epsilon} .
$$

Remarks. We may assume $q \leq N$, else the result is trivial. The restriction $|\beta| \leq$ $1 / q^{2}$ may be dropped but this is unimportant. We have not tried to replace the $N^{\epsilon}$ by a power of $\log N$ in this theorem. It seems highly likely that this can be done and that this would lead to the replacement of $n^{\epsilon}$ by a power of $\log n$ in Theorem 1.

The estimates (1.17) and (1.18) are stronger than Vaughan's estimate (1.16) in certain ranges. If we write $q=N^{\nu}$ and choose $T_{0}$ bounded (smaller ranges otherwise apply) then (1.17) is stronger than (1.16) in the range $2 / 5<\nu<9 / 20$ while (1.18) is stronger than (1.16) for $2 / 5<\nu<1 / 2$. For both (1.17) and (1.18) the best exponent is $19 / 24=4 / 5-1 / 120$ (which occurs at $\nu=5 / 12$ ) so that the improvements are small. Actually the (a) part of this theorem is incidental to this work; it is only the (b) part that is needed. We include the former since it comes with no extra work (and because Vaughan's refinement of the Vinogradov estimate is so well known).

The estimate (1.19) uses amongst other things the fact, due to Huxley and Jutila [HJ], that the density estimate (1.7) holds for $\sigma>4 / 5$. It follows easily from the proof given below that one can refine (1.19) by using the improvement in HeathBrown [H-B] that the density estimate holds for $\sigma>15 / 19$.

The sum $r_{k}(n)$ can (see Section 3 ) be given explicitly in terms of the non-trivial zeros of the Dirichlet $L$-functions together with some error terms which are in many cases small. This formula is simplified in case we replace $r_{k}(n)$ by a sum weighted by the von Mangoldt function. We state this here in the simplest case.

Proposition 1. Let $k \geq 5$, and assume the Generalized Riemann Hypothesis. Then

$$
\begin{aligned}
& \sum_{n_{1}+n_{2}+\cdots+n_{k}=n} \Lambda\left(n_{1}\right) \Lambda\left(n_{2}\right) \cdots \Lambda\left(n_{k}\right) \\
& =\frac{n^{k-1}}{(k-1) !} \mathfrak{S}_{k}(n)-\frac{k}{(k-3) !} \sum_{\rho}+O\left(n^{k-7 / 4+\epsilon}\right),
\end{aligned}
$$

with

$$
\sum_{\rho}=\sum_{q=1}^{\infty} \frac{\mu^{k-1}(q)}{\phi^{k}(q)} \sum_{\chi(q)} \sum_{h(q)} \bar{\chi}(h) c_{q}(h-n) \sum_{\rho(\chi)} \frac{n^{\rho+k-2}}{\rho} B(\rho+1, k-2),
$$

where $B$ is the Euler beta function, given for Re $u>0$, Re $v>0$, by

$$
B(u, v)=\int_{0}^{1} t^{u-1}(1-t)^{v-1} d t
$$

and $\rho(\chi)$ runs through the non-trivial zeros of $L(s, \chi)$. In case $k \geq 6$, we can replace $7 / 4$ by 2 .

This result, together with the arguments in Sections 3 and 4, suggest the possibility that statements like (1.10) may be subject to substantial further refinements wherein main terms of lower order are explicitly given. 


\section{ACKNOWLEDGEMENTS}

The work on an early version of this paper was accomplished while J.F. was enjoying a Research Professorship at MSRI, Berkeley. The excellent working conditions were greatly appreciated. Thanks are due to A. Selberg for a suggestion which led us to a sharpening of the cases $k \geq 5$ of Theorems 2 and 3 and to the above explicit formula. We are also indebted to the referee for a careful reading of the paper and a number of interesting remarks.

\section{Notation and Some Lemmas}

We first need to introduce some of the notation of [Go]. We define

$$
S(\alpha)=\sum_{p \leq N}(\log p) e(p \alpha),
$$

and therefore have

$$
\begin{gathered}
S^{k}(\alpha)=\sum_{n \leq k N} R_{k}(n, N) e(n \alpha), \\
R_{k}(n, N)=\sum_{\substack{p_{1}, p_{2}, \cdots, p_{k} \leq N \\
p_{1}+p_{2}+\cdots+p_{k}=n}}\left(\log p_{1}\right)\left(\log p_{2}\right) \cdots\left(\log p_{k}\right) .
\end{gathered}
$$

The appropriate approximation for $S(a / q+\beta)$, when $(a, q)=1$, is given by

$$
J(\beta)=\frac{\mu(q)}{\phi(q)} I(\beta), \quad I(\beta)=\sum_{1 \leq n \leq N} e(n \beta),
$$

and we have

$$
I^{k}(\alpha)=\sum_{n \leq k N} \nu_{k}(n, N) e(n \alpha), \quad \nu_{k}(n, N)=\sum_{\substack{1 \leq n_{1}, n_{2}, \cdots, n_{k} \leq N \\ n_{1}+n_{2}+\cdots+n_{k}=n}} 1 .
$$

Next, the approximation for $S^{k}(\alpha)$ for all $\alpha$ is given by

$$
V_{k}(\alpha)=\sum_{n \leq k N} \mathfrak{S}_{k}(n) \nu_{k}(n, N) e(n \alpha)
$$

where $\mathfrak{S}_{k}(n)$ is given by $(1.2)$.

We define the Farey fractions of order $Q$ where, throughout the paper, we take $1 \leq Q \leq N$. These are : $\{a / q: 1 \leq q \leq Q, 0 \leq a \leq q,(a, q)=1\}$. The Farey arcs around each of these fractions, except $0 / 1$ which we exclude, are defined as follows. Let $a^{\prime} / q^{\prime}<a / q<a^{\prime \prime} / q^{\prime \prime}$ be consecutive fractions in the Farey decomposition of order $Q$, and for $a \neq 0$ let

$$
\begin{aligned}
& \mathcal{M}_{Q}(q, a)=\left(\frac{a+a^{\prime}}{q+q^{\prime}}, \frac{a+a^{\prime \prime}}{q+q^{\prime \prime}}\right]=\left(\frac{a}{q}-\frac{1}{q\left(q+q^{\prime}\right)}, \frac{a}{q}+\frac{1}{q\left(q+q^{\prime \prime}\right)}\right], \text { for } \frac{a}{q} \neq \frac{1}{1} \\
& \mathcal{M}_{Q}(1,1)=\left(1-\frac{1}{Q+1}, 1+\frac{1}{Q+1}\right] .
\end{aligned}
$$


These intervals are disjoint and their union covers the interval $\left(\frac{1}{Q+1}, 1+\frac{1}{Q+1}\right]$. We denote the Farey arcs re-centered to the origin by $\theta_{Q}(q, a)$ :

$$
\theta_{Q}(q, a)=\left(\frac{-1}{q\left(q+q^{\prime}\right)}, \frac{1}{q\left(q+q^{\prime \prime}\right)}\right]
$$

when $q \neq 1$, and $\theta_{Q}(1,1)=\left(\frac{-1}{Q+1}, \frac{1}{Q+1}\right]$. We shall use on occasion the relation

$$
\left(\frac{-1}{2 q Q}, \frac{1}{2 q Q}\right) \subseteq \theta_{Q}(q, a) \subseteq\left(\frac{-1}{q Q}, \frac{1}{q Q}\right) .
$$

It is very convenient in many cases to abbreviate the above notation by

$$
S=S(a / q+\beta), \quad J=J(\beta), \quad I=I(\beta), \quad V_{k}=V_{k}(a / q+\beta), \quad \theta=\theta_{Q}(q, a) ;
$$

and denote a sum over all Farey fractions of order $Q$ by

$$
\sum_{Q}=\sum_{1 \leq q \leq Q} \sum_{\substack{1 \leq a \leq q \\(a, q)=1}}
$$

Thus for example we write

$$
\sum_{1 \leq q \leq Q} \sum_{\substack{1 \leq a \leq q \\(a, q)=1}} \int_{\theta_{Q}(q, a)}\left|S^{k}(a / q+\beta)-V_{k}(a / q+\beta)\right|^{2} d \beta=\sum_{Q} \int_{\theta}\left|S^{k}-V_{k}\right|^{2} .
$$

We need to recall a few results from $[\mathrm{Go}]$.

Lemma 1. We have, for $1 \leq Q \leq N$,

$$
\begin{gathered}
\int_{0}^{1}|S(\alpha)|^{2} d \alpha \sim N \log N, \\
\sum_{Q} \int_{\theta}|J|^{2} \ll N \log N,
\end{gathered}
$$

and

$$
\sum_{Q} \int_{\theta}|S-J|^{2} \ll N \log N
$$

Proof. Equation (2.1) follows from Parseval's theorem and the prime number theorem. The expression in (2.2) is dominated by

$$
\sum_{1 \leq q \leq Q} \frac{1}{\phi(q)} \int_{0}^{1}|I(\beta)|^{2} d \beta=N(\log Q+O(1))
$$

by Parseval and [Go, Lemma 2], and (2.2) follows. Equation (2.3) is contained in [Go, Lemma 6], but also follows immediately by Cauchy-Schwarz from (2.1) and (2.2).

Lemma 2. Assuming GRH, we have

$$
\sum_{Q} \int_{\theta}|S-J|^{2}|J|^{2} \ll N^{2}(\log N)^{5}
$$

and for any real $c>0$,

$$
\sum_{Q} \frac{1}{\phi^{c}(q)} \int_{\theta}|S-J|^{2}|J|^{2} \ll N^{2}(\log N)^{4} .
$$


Proof. This is just the special case $\Theta=\frac{1}{2}$ of Lemma 5 in Section 7 and we postpone the proof until then.

To estimate higher powers of $S$ and $J$ integrated over Farey arcs we need some pointwise uniform estimates for $S_{1}(\beta, q, a)=S-J$ on all Farey arcs. We therefore define

$$
S^{*}(Q)=\max _{1 \leq q \leq Q} \max _{\substack{1 \leq a \leq q \\(a, q)=1}} \max _{\beta \in \theta}|S-J|, \quad \mathcal{S}_{*}=\min _{1 \leq Q \leq N} S^{*}(Q) .
$$

Thus by (1.15) we have on GRH, since $|\beta| \leq 1 / q Q$,

$$
S^{*}(Q) \ll Q^{1 / 2} N^{1 / 2}(\log (Q N))^{2}+Q^{-1 / 2} N \log (Q N),
$$

and therefore $\mathcal{S}_{*} \ll N^{3 / 4}(\log N)^{3 / 2}$ which occurs when $Q=N^{1 / 2} / \log N$. We make the conjecture, letting $Q=N^{\eta}$, that

$$
S^{*}(Q) \ll N^{\max (\eta, 1-\eta)+\epsilon},
$$

and therefore $\mathcal{S}_{*} \ll N^{1 / 2+\epsilon}$.

Lemma 3. Let $m \geq 2$. We have

$$
\sum_{Q} \int_{\theta}|S-J|^{m} \ll\left(S^{*}(Q)\right)^{m-2} N \log N
$$

Assuming GRH the following also hold:

$$
\begin{gathered}
\sum_{Q} \int_{\theta}|S-J|^{m}|J| \ll\left(S^{*}(Q)\right)^{m-2} N^{3 / 2}(\log N)^{3}, \\
\sum_{Q} \int_{\theta}|S-J|^{m}|J|^{2} \ll\left(S^{*}(Q)\right)^{m-2} N^{2}(\log N)^{5}, \\
\sum_{Q} \int_{\theta}|S-J||J|^{2} \ll N^{3 / 2}(\log N)^{3},
\end{gathered}
$$

and, for $r \geq 3$,

$$
\sum_{Q} \int_{\theta}|S-J|^{m}|J|^{r} \ll\left(S^{*}(Q)\right)^{m-2} N^{r}(\log N)^{4}
$$

and

$$
\sum_{Q} \int_{\theta}|S-J||J|^{r} \ll N^{r-1 / 2}(\log N)^{5 / 2}
$$

Proof. The left hand of (2.9) is

$$
\ll\left(S^{*}(Q)\right)^{m-2} \sum_{Q} \int_{\theta}|S-J|^{2}
$$


and (2.9) follows from (2.3). By Cauchy-Schwarz the left hand side of (2.10) is

$$
\begin{aligned}
& \ll\left(S^{*}(Q)\right)^{m-2} \sum_{Q} \int_{\theta}(|S-J||J|)|S-J| \\
& \ll\left(S^{*}(Q)\right)^{m-2}\left(\sum_{Q} \int_{\theta}|S-J|^{2}|J|^{2}\right)^{1 / 2}\left(\sum_{Q} \int_{\theta}|S-J|^{2}\right)^{1 / 2},
\end{aligned}
$$

and (2.10) follows from (2.4) and (2.3). The left hand side of (2.13) (or (2.11) when $r=2)$ is

$$
\ll N^{r-2}\left(S^{*}(Q)\right)^{m-2} \sum_{Q} \frac{1}{\phi^{r-2}(q)} \int_{\theta}|S-J|^{2}|J|^{2}
$$

and (2.11) follows from (2.4) and (2.13) follows from (2.5). Finally, the left hand side of (2.14) (or (2.12) when $r=2$ ) is, again by the Cauchy-Schwarz inequality,

$$
\begin{aligned}
& \ll N^{r-2} \sum_{Q} \frac{1}{\phi^{r-2}(q)} \int_{\theta}|S-J||J|^{2} \\
& \ll N^{r-2}\left(\sum_{Q} \int_{\theta}|J|^{2}\right)^{1 / 2}\left(\sum_{Q} \frac{1}{\phi^{2(r-2)}(q)} \int_{\theta}|S-J|^{2}|J|^{2}\right)^{1 / 2},
\end{aligned}
$$

and, using (2.2), we see that (2.12) follows from (2.4), and (2.14) follows from $(2.5)$.

\section{An Explicit Formula FOR $r_{k}(n)$}

In this section we give the argument for an explicit formula which expresses $r_{k}(n)$ in terms of the non-trivial zeros of the Dirichlet $L$-functions and the somewhat cleaner formula (stated in the introduction) which holds for the corresponding sum in which the representations of $n$ as a sum are counted with weight $\Lambda\left(n_{j}\right)$ rather than $\log p_{j}$. As throughout the paper, implicit constants may depend on $k$, but we shall not indicate this. By the Fourier coefficient formula

$$
R_{k}(n, N)=\int_{0}^{1} S^{k}(\alpha) e(-n \alpha) d \alpha
$$

We take $N=n$, and therefore have $r_{k}(n)=R_{k}(n, N)$. By the binomial theorem

$$
S^{k}=((S-J)+J)^{k}=\sum_{m=0}^{k}\left(\begin{array}{c}
k \\
m
\end{array}\right)(S-J)^{m} J^{k-m},
$$

and thus

$$
\begin{aligned}
r_{k}(n) & =\sum_{Q} e\left(\frac{-n a}{q}\right) \int_{\theta} S^{k}\left(\frac{a}{q}+\beta\right) e(-n \beta) d \beta \\
& =M_{0}(k)+k M_{1}(k)+\sum_{m=2}^{k} O\left(E_{m}\right)
\end{aligned}
$$

where

$$
M_{m}(k)=\sum_{Q} e\left(\frac{-n a}{q}\right) \int_{\theta}\left(S\left(\frac{a}{q}+\beta\right)-J(\beta)\right)^{m} J^{k-m}(\beta) e(-n \beta) d \beta
$$


satisfies $\left|M_{m}(k)\right| \leq E_{m}(k)$ with

$$
E_{m}=E_{m}(k)=\sum_{Q} \int_{\theta}|S-J|^{m}|J|^{k-m} .
$$

We first deal with the main terms $M_{0}(k)$ and $M_{1}(k)$ in the following lemma.

Lemma 4. Let $N=n, k \geq 3$, and $n^{\epsilon} \leq Q \leq n$. Then we have

$$
M_{0}(k)=\frac{n^{k-1}}{(k-1) !} \mathfrak{S}_{k}(n)+O\left(n^{k-2}\right)+O\left(Q^{k}\right)+O\left(\frac{n^{k-1+\epsilon}}{Q^{k-1}}\right),
$$

and

$$
\begin{gathered}
M_{1}(k)=-\frac{1}{(k-3) !} \sum_{Q} \frac{\mu^{k-1}(q)}{\phi^{k}(q)} e\left(\frac{-n a}{q}\right) \sum_{\chi(q)} \tau(a, \bar{\chi}) \sum_{\rho(\chi)} \frac{n^{\rho+k-2}}{\rho} B(\rho+1, k-2) \\
+O\left(n^{k-3 / 2}+n^{3 / 2} \log Q+n Q^{k-1}\right) .
\end{gathered}
$$

Here $\tau$ is the Gauss sum $\tau(a, \chi)=\sum_{h(q)} e(h a / q) \chi(h), B$ denotes the beta function and $\rho$ runs through the non-trivial zeros of $L(s, \chi)$. This last sum is, as we shall see, absolutely convergent and by a simple rearrangement of the order of summation the above can be put in the form occurring in Proposition 1. We remark that the sum over $q$ in (3.4) may be extended to all $q$ (which has been done in (3.3)) at the cost of an additional error $O\left(n^{k-2+\Theta} Q^{3-k+\epsilon}\right)$ which is admissible in case $k \geq 4$. For the particular choice $Q=n^{1 / 2} / \log n$ we thus have, in case $k \geq 4$,

$$
M_{1}(k) \ll n^{k-2+\Theta} .
$$

The explicit formula arises when we combine in (3.2) the results of Lemma 4 with the estimates for the $E_{m}(k)$ to follow. In case $k \geq 5$ (or in case $k=4, \Theta>3 / 5$ ) these terms are small compared to the contribution coming from the sum over the zeros. In these cases the errors are all $o\left(n^{k-2+\Theta}\right)$ except in case $\Theta=1 / 2$ where the term $O\left(n^{k-3 / 2}\right)$ from (3.4) comes into play. This term arises from the contribution from squares of primes and could be made explicit. It is in any case not present for the corresponding sum where $r_{k}(n)$ is weighted by the von Mangoldt function.

Proof of Lemma 4. To obtain (3.3), we first note

$$
\begin{aligned}
\int_{\theta} J^{k}(\beta) e(-n \beta) d \beta & =\int_{0}^{1} J^{k}(\beta) e(-n \beta) d \beta+O\left(\frac{\mu^{2}(q)}{\phi^{k}(q)} \int_{1 / 2 q Q}^{1 / 2}|\beta|^{-k} d \beta\right) \\
& =\frac{\mu^{k}(q)}{\phi^{k}(q)}\left(\nu_{k}(n, N)+O(q Q)^{k-1}\right) .
\end{aligned}
$$

Since for $N=n$,

$$
\nu_{k}(n, N)=\nu_{k}(n)=\sum_{n_{1}+n_{2}+\cdots+n_{k}=n} 1=\frac{n^{k-1}}{(k-1) !}+O\left(n^{k-2}\right),
$$


we have

$$
\begin{aligned}
M_{0}(k)= & \left(\sum_{1 \leq q \leq Q} \frac{\mu^{k}(q)}{\phi^{k}(q)} c_{q}(-n)\right)\left(\frac{n^{k-1}}{(k-1) !}+O\left(n^{k-2}\right)\right) \\
& +O\left(Q^{k-1} \sum_{q \leq Q} \frac{\mu^{2}(q)}{\phi^{k-1}(q)} q^{k-1}\right) \\
= & \frac{n^{k-1}}{(k-1) !}\left(\sum_{1 \leq q \leq Q} \frac{\mu^{k}(q)}{\phi^{k}(q)} c_{q}(-n)\right)+O\left(n^{k-2}\right)+O\left(Q^{k}\right) .
\end{aligned}
$$

Now, extending the first sum to all $q$, we introduce an error

$$
\begin{aligned}
\sum_{q>Q} \frac{\mu^{2}(q)}{\phi^{k}(q)}\left|c_{q}(-n)\right| & \leq \sum_{q>Q} \frac{\mu^{2}(q)}{\phi^{k}(q)} \sum_{\substack{d|n \\
d| q}} d \leq \sum_{d \mid n} \frac{d \mu^{2}(d)}{\phi^{k}(d)} \sum_{q^{\prime}>Q / d} \frac{\mu^{2}\left(q^{\prime}\right)}{\phi^{k}\left(q^{\prime}\right)} \\
& \ll \frac{1}{Q^{k-1}} \sum_{d \mid n} \frac{d^{k}}{\phi^{k}(d)} \ll \frac{n^{\epsilon}}{Q^{k-1}},
\end{aligned}
$$

and (3.3) follows.

Turning to $M_{1}(k)$, we first note (again with $N=n$ )

$$
\begin{aligned}
\int_{\theta} S J^{k-1} e(-n \beta) d \beta=\sum_{p \leq n}(\log p) e(p a / q) \int_{\theta} J^{k-1}(\beta) e((p-n) \beta) d \beta \\
=\sum_{p \leq n}(\log p) e(p a / q) \int_{0}^{1} J^{k-1}(\beta) e((p-n) \beta) d \beta+O\left(\frac{n \mu^{2}(q)(q Q)^{k-2}}{\phi^{k-1}(q)}\right) \\
=\frac{\mu^{k-1}(q)}{\phi^{k-1}(q)} \sum_{p \leq n}(\log p) e(p a / q) \nu_{k-1}(n-p)+O\left(\frac{n \mu^{2}(q)(q Q)^{k-2}}{\phi^{k-1}(q)}\right) \\
=\frac{1}{(k-2) !} \frac{\mu^{k-1}(q)}{\phi^{k-1}(q)} \sum_{p \leq n}(\log p) e(p a / q)(n-p)^{k-2} \\
\quad+O\left(\frac{\mu^{2}(q)}{\phi^{k-1}(q)}\left(n^{k-2}+n(q Q)^{k-2}\right)\right)
\end{aligned}
$$

Now,

$$
\begin{aligned}
\sum_{p \leq n}(\log p) e(p a / q)(n-p)^{k-2}= & \sum_{(b, q)=1} e(b a / q) \sum_{\substack{p \leq n \\
p \equiv b(q)}}(\log p)(n-p)^{k-2} \\
& +O\left(n^{k-2} \log q\right) .
\end{aligned}
$$


For $(b, q)=1$,

$$
\begin{aligned}
\sum_{\substack{p \leq n \\
p \equiv b(q)}}(\log p)(n-p)^{k-2}= & \int_{0}^{n}(n-t)^{k-2} d(\theta(t ; q, b)) \\
= & \frac{1}{\phi(q)} \frac{n^{k-1}}{(k-1)}+(k-2) \int_{0}^{n}\left(\theta(t ; q, b)-\frac{t}{\phi(q)}\right)(n-t)^{k-3} d t \\
= & \frac{1}{\phi(q)} \frac{n^{k-1}}{(k-1)}-\frac{(k-2)}{\phi(q)} \sum_{\chi(q)} \bar{\chi}(b) \sum_{\rho(\chi)} \frac{1}{\rho} \int_{0}^{n} t^{\rho}(n-t)^{k-3} d t \\
& \quad O\left(n^{\epsilon}\right)+O\left(n^{k-2} \sum_{\substack{p^{j} \leq n \\
j \geq 2 \\
p^{j} \equiv b(q)}} \log p\right) .
\end{aligned}
$$

Substituting this in, we see that the left hand side of (3.9) is

$$
\frac{\mu(q)}{\phi(q)} \frac{n^{k-1}}{(k-1)}-\frac{(k-2)}{\phi(q)} \sum_{\chi(q)} \tau(a, \bar{\chi}) \sum_{\rho(\chi)} \frac{n^{\rho+k-2}}{\rho} B(\rho+1, k-2)+O\left(n^{k-3 / 2}\right)
$$

where, for $\operatorname{Re} u>0, \operatorname{Re} v>0$,

$$
B(u, v)=\int_{0}^{1} t^{u-1}(1-t)^{v-1} d t
$$

is the beta function. Since $B(u, v)=\Gamma(u) \Gamma(v) / \Gamma(u+v)$ and $\Gamma(s+1)=s \Gamma(s)$, we have $B(\rho+1, k-2) \ll|\Im \rho|^{2-k}$, so that the above sum over $\rho$ is absolutely convergent for $k \geq 3$. Substituting (3.10) in (3.8), we find that the left hand side of the latter is given by

$$
\begin{aligned}
\frac{\mu^{k}(q)}{\phi^{k}(q)} \frac{n^{k-1}}{(k-1) !}- & \frac{1}{(k-3) !} \frac{\mu^{k-1}(q)}{\phi^{k}(q)} \sum_{\chi(q)} \tau(a, \bar{\chi}) \sum_{\rho(\chi)} \frac{n^{\rho+k-2}}{\rho} B(\rho+1, k-2) \\
& +O\left(\frac{n^{k-3 / 2}}{\phi^{k-1}(q)}\right)+O\left(\frac{\mu^{2}(q)}{\phi^{k-1}(q)} n(q Q)^{k-2}\right) .
\end{aligned}
$$

We twist this by $e(-n a / q)$, and sum over $a$, then $q$. From the trivial identity $(S-J) J^{k-1}=-J^{k}+S J^{k-1}$, we deduce that

$$
M_{1}(k)=-M_{0}(k)+\sum_{Q} e(-n a / q) \int_{\theta} S J^{k-1} e(-n \beta) d \beta,
$$

so that (3.6) and (3.11) give (3.4).

\section{Proof of Theorem 2}

We take $Q=N^{1 / 2} / \log N$ and obtain by $(2.7)$ that $S^{*}(Q) \ll N^{3 / 4}(\log N)^{3 / 2}$. We take $N=n$. Since $\Theta=1 / 2$, we have by (3.5),

$$
M_{1}(k) \ll n^{k-3 / 2}
$$

in case $k \geq 4$, while for $k=3$ we have by (2.14),

$$
M_{1}(3) \ll E_{1}(3) \ll n^{3 / 2}(\log n)^{5 / 2} .
$$


For $2 \leq m \leq k-2$, by $(2.11)$ and (2.13),

$$
E_{m} \ll N^{k-m}\left(N^{3 / 4}(\log N)^{3 / 2}\right)^{m-2}(\log N)^{5} \ll N^{k-3 / 2-m / 4}(\log N)^{3 m / 2+2},
$$

and hence $E_{m}=o\left(N^{k-3 / 2}\right)$. By (2.10), taking $m=k-1$,

$$
E_{k-1} \ll\left(N^{3 / 4}(\log N)^{3 / 2}\right)^{k-3} N^{3 / 2}(\log N)^{3} \ll N^{3 k / 4-3 / 4}(\log N)^{3 k / 2-3 / 2},
$$

so that $E_{k-1}(k)=o\left(N^{k-3 / 2}\right)$ if $k \geq 4$ and $E_{2}(3)=O\left(N^{3 / 2}(\log N)^{3}\right)$. Finally, by $(2.9)$,

$$
E_{k} \ll\left(N^{3 / 4}(\log N)^{3 / 2}\right)^{k-2} N \log N \ll N^{3 k / 4-1 / 2}(\log N)^{3 k / 2-2} .
$$

Thus we find that, for $k \geq 5, E_{k}(k)=o\left(N^{k-3 / 2}\right)$, while $E_{4}(4) \ll N^{5 / 2}(\log N)^{4}$ and $E_{3}(3) \ll N^{7 / 4}(\log N)^{5 / 2}$. If we assume in addition the conjecture (2.8), we see that $E_{3}(3) \ll N^{3 / 2+\epsilon}$. Theorem 2 now follows from (3.2) and Lemma 4.

\section{Proof of Theorem 3}

The proof we give here applies to general $k \geq 3$ but, in case $k \geq 5$, is weaker than that which follows immediately from Theorem 2. By Parseval's theorem we have

$$
\sum_{n \leq k N}\left(R_{k}(n, N)-\nu_{k}(n, N) \mathfrak{S}_{k}(n)\right)^{2}=\int_{0}^{1}\left|S^{k}(\alpha)-V_{k}(\alpha)\right|^{2} d \alpha .
$$

Since for $1 \leq n \leq N$ we have $\nu_{k}(n, N)=n^{k-1} /(k-1) !+O\left(n^{k-2}\right)$, it follows by positivity that the left hand side of (5.1) is

$$
\sum_{n \leq k N} \geq \sum_{n \leq N}=\sum_{n \leq N}\left(r_{k}(n)-\frac{n^{k-1}}{(k-1) !} \mathfrak{S}_{k}(n)\right)^{2}+O\left(N^{2 k-2}\right),
$$

and therefore, to prove Theorem 3 it suffices to bound the right hand side of (5.1). To do this we use the Farey decomposition and find by the Cauchy-Schwarz inequality that

$$
\begin{aligned}
\int_{0}^{1}\left|S^{k}(\alpha)-V_{k}(\alpha)\right|^{2} d \alpha & =\sum_{Q} \int_{\theta}\left|S^{k}-V_{k}\right|^{2} \\
& \ll \sum_{Q} \int_{\theta}\left|S^{k}-J^{k}\right|^{2}+\sum_{Q} \int_{\theta}\left|V_{k}-J^{k}\right|^{2}=I_{1}+I_{2} .
\end{aligned}
$$

To estimate $I_{1}$ we use (3.1) and the estimate $\left|\sum_{m=1}^{k} a_{m}\right|^{2} \leq k \sum_{m=1}^{k}\left|a_{m}\right|^{2}$ to see that, for $k \geq 3$,

$$
I_{1} \ll \sum_{m=1}^{k} \sum_{Q} \int_{\theta}|S-J|^{2 m}|J|^{2(k-m)}=\sum_{m=1}^{k} S_{m} .
$$

We again choose $Q=N^{1 / 2} / \log N$. By (2.13), $S_{1} \ll N^{2 k-2}(\log N)^{4}$. By $(2.11)$ and $(2.13)$,

$$
\begin{aligned}
S_{m} & \ll N^{2(k-m)}\left(N^{3 / 4}(\log N)^{3 / 2}\right)^{2 m-2}(\log N)^{5} \\
& \ll N^{2 k-m / 2-3 / 2}(\log N)^{3 m+2}=o\left(N^{2 k-2}\right),
\end{aligned}
$$

for $2 \leq m \leq k-1$. Finally, by (2.9),

$$
S_{k} \ll\left(N^{3 / 4}(\log N)^{3 / 2}\right)^{2 k-2} N \log N \ll N^{3 k / 2-1 / 2}(\log N)^{3 k-2},
$$


and therefore $S_{k} \ll N^{4}(\log N)^{7}$ for $k=3$ and $S_{k}=o\left(N^{2 k-2}\right)$ for $k \geq 4$. We conclude that in general, for $k \geq 3$,

$$
I_{1} \ll \max \left(N^{2 k-2}(\log N)^{4}, N^{4}(\log N)^{7}\right) .
$$

We now show that, for $Q=N^{1 / 2} / \log N$ and $k \geq 3$,

$$
I_{2} \ll N^{k+\epsilon}=o\left(N^{2 k-2}\right),
$$

and therefore Theorem 3 follows from (5.1), (5.2), and (5.3).

To prove (5.3) we first truncate the singular series at $Q$ and use (3.7) to find

$$
\mathfrak{S}_{k}(n)=\sum_{q \leq Q} \frac{\mu^{k}(q)}{\phi^{k}(q)} c_{q}(-n)+O\left(\frac{n^{\epsilon}}{Q^{k-1}}\right)=\mathfrak{S}_{k}(n, Q)+O\left(\frac{n^{\epsilon}}{Q^{k-1}}\right) .
$$

Writing

$$
V_{k}(\alpha, Q)=\sum_{n \leq k N} \mathfrak{S}_{k}(n, Q) \nu_{k}(n, N) e(n \alpha)
$$

we have

$$
I_{2} \ll \sum_{Q} \int_{\theta}\left|V_{k}(a / q+\beta, Q)-J^{k}\right|^{2}+\int_{0}^{1}\left|V_{k}(\alpha)-V_{k}(\alpha, Q)\right|^{2} d \alpha .
$$

The second integral in (5.5) is, by Parseval and (5.4),

$$
\begin{aligned}
& =\sum_{n \leq k N}\left|\left(\mathfrak{S}_{k}(n)-\mathfrak{S}_{k}(n, Q)\right) \nu_{k}(n, N)\right|^{2} \\
& \ll \sum_{n \leq k N}\left(\frac{n^{\epsilon}}{Q^{k-1}} n^{k-1}\right)^{2} \ll \frac{N^{2 k-1+\epsilon}}{Q^{2 k-2}} \ll N^{k+\epsilon},
\end{aligned}
$$

since $Q=N^{1 / 2} / \log N$. Next,

$$
\begin{aligned}
& \sum_{Q} \int_{\theta}\left|V_{k}(a / q+\beta, Q)-J^{k}\right|^{2} \\
& =\sum_{Q} \int_{\theta}\left|\sum_{r \leq Q} \frac{\mu^{k}(r)}{\phi^{k}(r)} \sum_{\substack{1 \leq b \leq r,(b, r)=1 \\
\text { bo } \frac{b}{r} \neq \frac{a}{q}}} I^{k}\left(\frac{a}{q}-\frac{b}{r}+\beta\right)\right|^{2} d \beta \\
& =\sum_{r, r^{\prime} \leq Q} \frac{\mu^{k}(r)}{\phi^{k}(r)} \frac{\mu^{k}\left(r^{\prime}\right)}{\phi^{k}\left(r^{\prime}\right)} \sum_{\substack{1 \leq b \leq r,(b, r)=1 \\
1 \leq b^{\prime} \leq r^{\prime},\left(b^{\prime}, r^{\prime}\right)=1}} \int_{E} I^{k}\left(\beta-\frac{b}{r}\right) \overline{I^{k}\left(\beta-\frac{b^{\prime}}{r^{\prime}}\right)} d \beta,
\end{aligned}
$$

where $E=[0,1] \backslash\left(\mathcal{M}_{Q}(r, b) \cup \mathcal{M}_{Q}\left(r^{\prime}, b^{\prime}\right)\right)$. We break this last sum into two sums $S_{1}$ and $S_{2}$ where

$$
S_{1}=\sum_{\frac{b}{r}=\frac{b^{\prime}}{r^{\prime}}}, \quad S_{2}=\sum_{\frac{b}{r} \neq \frac{b^{\prime}}{r^{\prime}}}
$$


Now

$$
\begin{aligned}
S_{1} & =\sum_{r \leq Q} \frac{\mu^{2}(r)}{\phi^{2 k}(r)} \sum_{\substack{1 \leq b \leq r \\
(b, r)=1}} \int_{[0,1] \backslash \mathcal{M}_{Q}(r, b)}\left|I^{k}\left(\beta-\frac{b}{r}\right)\right|^{2} d \beta \\
& \ll \sum_{r \leq Q} \frac{1}{\phi^{2 k-1}(r)} \int_{1 / r Q}^{1 / 2} \frac{1}{\beta^{2 k}} d \beta \\
& \ll \sum_{r \leq Q}\left(\frac{r Q}{\phi(r)}\right)^{2 k-1} \ll Q^{2 k} \ll N^{k} .
\end{aligned}
$$

For $S_{2}$ we may by symmetry restrict attention to the terms $b / r<b^{\prime} / r^{\prime}$; these contribute

$$
\ll \sum_{r, r^{\prime} \leq Q} \frac{\mu^{2}(r) \mu^{2}\left(r^{\prime}\right)}{\phi^{k}(r) \phi^{k}\left(r^{\prime}\right)} \sum_{\substack{1 \leq b \leq r,(b, r)=1 \\ 1 \leq b^{\prime} \leq r^{\prime},\left(b^{\prime}, r^{\prime}\right)=1 \\ b r^{\prime}<b^{\prime} r}} \int_{I} \frac{d \beta}{\left\|\beta-\frac{b}{r}\right\|^{k}\left\|\beta-\frac{b^{\prime}}{r^{\prime}}\right\|^{k}},
$$

where

$$
\int_{I}=\left\{\int_{\frac{1}{Q}}^{\frac{b}{r}-\frac{1}{r Q}}+\int_{\frac{b}{r}+\frac{1}{r Q}}^{\frac{b^{\prime}}{r^{\prime}}-\frac{1}{r^{\prime} Q}}+\int_{\frac{b^{\prime}}{r^{\prime}}+\frac{1}{r^{\prime} Q}}^{1+\frac{1}{Q}}\right\} .
$$

If $b / r$ and $b^{\prime} / r^{\prime}$ are consecutive Farey fractions of order $Q$ then the middle integral does not occur. Using the estimate

$$
\begin{aligned}
\left(\frac{1}{(x-a)(x-b)}\right)^{k} & =\left(\frac{1}{a-b}\left(\frac{1}{x-a}-\frac{1}{x-b}\right)\right)^{k} \\
& \ll \frac{1}{(a-b)^{k}}\left(\frac{1}{(x-a)^{k}}+\frac{1}{(x-b)^{k}}\right),
\end{aligned}
$$

we can bound the integrals above and find

$$
\begin{aligned}
S_{2} & \ll \sum_{r, r^{\prime} \leq Q} \frac{\mu^{2}(r)}{\phi^{k}(r)} \frac{\mu^{2}\left(r^{\prime}\right)}{\phi^{k}\left(r^{\prime}\right)} \sum_{\substack{1 \leq b \leq r,(b, r)=1 \\
1 \leq b^{\prime} \leq r^{\prime},\left(b^{\prime}, r^{\prime}\right)=1 \\
b r^{\prime} \neq b^{\prime} r}} \frac{\left(r^{k-1}+r^{\prime k-1}\right) Q^{k-1}}{\left|b / r-b^{\prime} / r^{\prime}\right|^{k}} \\
& \ll \sum_{\substack{r, r^{\prime}, b, b^{\prime} \leq Q \\
b r^{\prime} \neq b^{\prime} r}}\left(\frac{r r^{\prime}}{\phi(r) \phi\left(r^{\prime}\right)}\right)^{k} \frac{\left(r^{k-1}+r^{\prime k-1}\right) Q^{k-1}}{\left|b r^{\prime}-b^{\prime} r\right|^{k}} .
\end{aligned}
$$

By the method of [Go, pp. 30-31] one can show this last expression is $\ll Q^{2 k}$, but we use here a simpler approach which suffices for our present purpose. Let $T_{n}(x)$ denote the number of ordered quadruples of positive integers $r, r^{\prime}, b$, and $b^{\prime}$ satisfying $n=b r^{\prime}-b^{\prime} r, b^{\prime} r \leq x$. The number of these for which $b^{\prime} r$ has a fixed value $\nu$ is $\tau(\nu) \tau(\nu+n)$, where $\tau$ is the divisor function. Ingham [In] gave the asymptotic formula

$$
T_{n}(x)=\sum_{1 \leq \nu \leq x} \tau(\nu) \tau(\nu+n) \sim \frac{6}{\pi^{2}} \sigma_{-1}(n) x(\log x)^{2}
$$


where $\sigma_{s}(n)=\sum_{d \mid n} d^{s}$. By following Ingham's argument it is easily seen that the upper bound $T_{n}(x) \ll \sigma_{-1}(n) x(\log x)^{2}$ holds uniformly for $0<|n| \leq 2 x$. Using this fact together with the estimate $r / \phi(r) \ll \log \log r$, we conclude

$$
\begin{aligned}
S_{2} & \ll Q^{2 k-2}(\log \log Q)^{2 k} \sum_{\substack{r, r^{\prime}, b, b^{\prime} \leq Q \\
b r^{\prime} \neq b^{\prime} r}} \frac{1}{\left|b r^{\prime}-b^{\prime} r\right|^{k}} \\
& \ll Q^{2 k-2}(\log \log Q)^{2 k} \sum_{0<|n| \leq 2 Q^{2}} \frac{1}{n^{k}} \sigma_{-1}(n) Q^{2}(\log Q)^{2} \\
& \ll Q^{2 k}(\log Q)^{3} .
\end{aligned}
$$

Thus we have $S_{2} \ll N^{k}$, and this completes the proof of (5.3).

\section{Proof of Theorem 4}

The method we use in this section is heavily based on [MV, Theorem 1] and we shall freely refer to the results of that paper. In the case $k=2$ a stronger bound is given in $[\mathrm{MV}]$ as a consequence of their Theorem 2 but, as already remarked, the corresponding strengthening cannot hold for arbitrary $k$.

We let $1 / 2 \leq \rho<1$ and $R=(1-\rho)^{-1}$. Let $H(z)=\sum_{p}(\log p) z^{p}$ so that we have $\sum_{n} r_{k}(n) z^{n}=H^{k}(z)$. As $\rho \rightarrow 1^{-}$we have by [MV, Lemma 5], $H(\rho) \sim R$ and $H(\rho)-R=\Omega\left(R^{1 / 2}\right)$. We deduce from the former that

$$
H^{k}(\rho)-R^{k}=(k+o(1))(H(\rho)-R) R^{k-1} .
$$

Also, by [MV, Lemma 3], we have

$$
\sum_{n=1}^{\infty} \mathfrak{S}_{k}(n) n^{k-1} \rho^{n}=(k-1) ! R^{k}+O\left(R^{k-1} \log R\right)
$$

and so the above facts give

$$
\sum_{n=1}^{\infty}\left(r_{k}(n)-\frac{n^{k-1}}{(k-1) !} \mathfrak{S}_{k}(n)\right) \rho^{n}=\Omega\left(R^{k-1 / 2}\right) .
$$

We now consider

$$
\begin{aligned}
A(\rho) & =\sum_{n=1}^{\infty} \sum_{m \leq n}\left|r_{k}(m)-\frac{m^{k-1}}{(k-1) !} \mathfrak{S}_{k}(m)\right| \rho^{n} \\
& =\sum_{m=1}^{\infty}\left|r_{k}(m)-\frac{m^{k-1}}{(k-1) !} \mathfrak{S}_{k}(m)\right| \sum_{j=m}^{\infty} \rho^{j} \\
& =R \sum_{m=1}^{\infty}\left|r_{k}(m)-\frac{m^{k-1}}{(k-1) !} \mathfrak{S}_{k}(m)\right| \rho^{m}
\end{aligned}
$$

which by (6.2) satisfies

$$
A(\rho)=\Omega\left(R^{k+1 / 2}\right)
$$

Now, assume that Theorem 4 is untrue so that we have

$$
\sum_{m \leq x}\left(r_{k}(m)-\frac{m^{k-1}}{(k-1) !} \mathfrak{S}_{k}(m)\right)^{2}=o\left(x^{2 k-2}\right) .
$$


By Cauchy's inequality this implies that

$$
\sum_{m \leq x}\left|r_{k}(m)-\frac{m^{k-1}}{(k-1) !} \mathfrak{S}_{k}(m)\right|=o\left(x^{k-1 / 2}\right) .
$$

This in turn yields

$$
A(\rho)=o\left(\sum_{n=1}^{\infty} n^{k-1 / 2} \rho^{n}\right) .
$$

But, by [MV, Lemma 2], this last sum is $\ll R^{k+1 / 2}$ so that $A(\rho)=o\left(R^{k+1 / 2}\right)$. This contradicts (6.3) thus completing the proof of Theorem 4.

\section{Preparations for Theorem 1}

In this section we give the proof of Theorem 5. Using some of the same ideas, we shall also prove Lemma 5 , a generalization of Lemma 2 . This more general form is needed for the cases $k \geq 4$ of Theorem 1 .

We begin by covering some ground common to both proofs. For $\chi \bmod q$ let

$$
\psi^{\prime}(x, \chi)=\sum_{n \leq x} \Lambda(n) \chi(n)-E(\chi) x,
$$

where $E(\chi)=1$ if $\chi=\chi_{0}$, the principal character, and is zero otherwise. We use the explicit formula in the form, valid for $x \geq 2, T \geq 2$, derived in [Oz] from [Da, p. 117, formulae (4),(5)],

$$
\psi^{\prime}(x, \chi)=-\sum_{\substack{\rho \\|\gamma| \leq T}} \frac{x^{\rho}}{\rho}+R_{1}(x)+R_{2}(x),
$$

where $\rho=b+i \gamma$ runs through the non-trivial zeros of $L(s, \chi)$ with $|\gamma| \leq T$, and where $R_{1}(x)=R_{1}(x, \chi)$ satisfies

$$
\int_{2^{-}}^{x}\left|d R_{1}(y)\right| \ll \log x
$$

while $R_{2}(x)=R_{2}(x, T, \chi)$ satisfies both

$$
R_{2}(x) \ll \log x+x T^{-1}(\log q x T)^{2},
$$

and

$$
\int_{2}^{x}\left|R_{2}(y)\right| d y \ll x^{2} T^{-1}(\log q x T)^{2} .
$$

Next let

$$
\Psi(N, \chi, \beta)=\sum_{n \leq N}^{\prime} \Lambda(n) \chi(n) e(n \beta)
$$

where $\sum^{\prime}$ denotes that, for $\chi=\chi_{0}, \sum^{\prime}=\sum_{n}-I(\beta)$. We have

$$
\Psi(N, \chi, \beta)=\int_{2^{-}}^{N} e(u \beta) d\left(\psi^{\prime}(u, \chi)\right),
$$


so that, inserting the explicit formula, and taking $T=N^{2}$, we obtain

$$
\begin{aligned}
\Psi(N, \chi, \beta) & =-\sum_{|\gamma| \leq N^{2}} \int_{2}^{N} e(u \beta) u^{\rho-1} d u+\int_{2^{-}}^{N} e(u \beta) d\left(R_{1}(u)+R_{2}(u)\right) \\
& =-\sum_{|\gamma| \leq N^{2}} \mathcal{I}_{\rho}+O\left((\log q N)^{2}\right),
\end{aligned}
$$

where

$$
\mathcal{I}_{\rho}=\int_{2}^{N} u^{b-1} e\left(u \beta+\frac{\gamma \log u}{2 \pi}\right) d u
$$

This integral may be estimated by results of van der Corput, cf. [Ti, Lemmata 4.3 and 4.5]. For $|\gamma| \leq 1+4 \pi|\beta| N$, say, we use the latter and find

$$
\mathcal{I}_{\rho} \ll N^{b}(1+|\gamma|)^{-1 / 2} .
$$

On the other hand, for $|\gamma|>1+4 \pi|\beta| N$, the former yields

$$
\mathcal{I}_{\rho} \ll N^{b}|\gamma|^{-1} \text {. }
$$

Proof of Theorem 5. The argument is based on that of Hardy-Littlewood for (1.15); compare with the more modern version of the latter in [BH, pp. 212-213]. Using instead a simpler argument due to Montgomery [Mo, Chapter 16] but incorporating therein the same density theorems of Huxley and Jutila used below, one can derive estimates of the same strength provided that $|\beta| \ll N^{-1}$ but apparently only somewhat weaker ones for larger $|\beta|$.

Let $\tau(\chi)=\sum_{n(q)} \chi(n) e(n / q)$, the Gaussian sum. Since we have $\tau\left(\chi_{0}\right)=\mu(q)$, $\sum_{p \mid q} \log p \leq \log q$, and $S(\alpha)=\sum_{n \leq N} \Lambda(n) e(n \alpha)+O\left(N^{1 / 2}\right)$, it suffices to prove the bounds claimed for $S_{1}$, instead for

$$
S_{2}(\beta ; q, a)=\frac{1}{\phi(q)} \sum_{\chi(q)} \chi(a) \tau(\bar{\chi}) \Psi(N, \chi, \beta) .
$$

Since $|\tau(\bar{\chi})| \leq q^{1 / 2}$, we have

$$
S_{2} \ll q^{-1 / 2+\epsilon} \sum_{\chi(q)}|\Psi(N, \chi, \beta)| .
$$

We estimate $\Psi$ using (7.2). The error term in (7.2) is admissible for the theorem and it suffices to consider $\sum_{\rho}$. Define

$$
Z(\sigma, T)=Z_{q}(\sigma, T)=\sum_{\chi(q)} N(\sigma, T, \chi) .
$$

By (7.4), (7.5), and partial summation we have

$$
\begin{gathered}
\sum_{\chi(q)}\left|\sum_{|\gamma| \leq N^{2}} \mathcal{I}_{\rho}\right| \ll_{\epsilon} N^{\epsilon}\left\{q\left(T_{0} N\right)^{1 / 2}+\max _{T \leq 4 \pi T_{0}} T^{-1 / 2} \int_{1 / 2}^{1} N^{\sigma} Z(\sigma, T) d \sigma\right. \\
\left.+\max _{T \geq T_{0}} T^{-1} \int_{1 / 2}^{1} N^{\sigma} Z(\sigma, T) d \sigma\right\} .
\end{gathered}
$$

To estimate the right side of (7.8) we shall use a number of density theorems. By a well-known result of Huxley [Hu] we have

$$
Z(\sigma, T) \ll_{\epsilon}(q T)^{12(1-\sigma) / 5+\epsilon} \quad \text { for } 1 / 2 \leq \sigma \leq 1 .
$$


We also have the Ingham-Montgomery estimates

$$
Z(\sigma, T) \ll_{\epsilon}(q T)^{(5-4 \sigma) / 3+\epsilon},
$$

valid for $1 / 2 \leq \sigma \leq 4 / 5$, and

$$
Z(\sigma, T) \ll_{\epsilon}(q T)^{(51-48 \sigma) / 25+\epsilon},
$$

valid for $1 / 2 \leq \sigma \leq 3 / 4$. These estimates are a simple consequence of [Mo, formula (12.9)]. To prove (1.17) we decompose the two integrals in (7.8) as

$$
\int_{1 / 2}^{11 / 16}+\int_{11 / 16}^{1}
$$

We apply (7.10) to the former and (7.9) to the latter. The contribution to the right hand side of (7.8) coming from the two integrals $\int_{1 / 2}^{11 / 16}$ is majorized as

$$
\begin{aligned}
& \ll_{\epsilon}\left\{\max _{\substack{T \leq 4 \pi T_{0} \\
T>N^{3 / 4} / q}} T^{-1 / 2}\left(N /(q T)^{4 / 3}\right)^{1 / 2}(q T)^{5 / 3}\right. \\
& +\max _{\substack{T \geq T_{0} \\
T>N^{3 / 4} / q}} T^{-1}\left(N /(q T)^{4 / 3}\right)^{1 / 2}(q T)^{5 / 3}+\max _{\substack{T \leq 4 \pi T_{0} \\
T \leq N^{3 / 4} / q}} T^{-1 / 2}\left(N /(q T)^{4 / 3}\right)^{11 / 16}(q T)^{5 / 3} \\
& \left.+\max _{\substack{T \geq T_{0} \\
T \leq N^{3 / 4} / q}} T^{-1}\left(N /(q T)^{4 / 3}\right)^{11 / 16}(q T)^{5 / 3}\right\} \\
& \ll_{\epsilon}\left\{q\left(T_{0} N\right)^{1 / 2}+q N^{1 / 2}+q^{3 / 4} T_{0}^{1 / 4} N^{11 / 16}+q^{3 / 4} N^{11 / 16}\right\} N^{\epsilon} .
\end{aligned}
$$

Similarly, the contribution to (7.8) from the integrals $\int_{11 / 16}^{1}$ is, by (7.9), majorized as

$$
\begin{aligned}
& \ll_{\epsilon}\left\{\max _{\substack{T \leq 4 \pi T_{0} \\
T>N^{5 / 12} / q}}+\max _{\substack{T>T_{0} \\
T>N^{5 / 12} / q}}+\max _{\substack{T \leq 4 \pi T_{0} \\
T \leq N^{5 / 12} / q}}+\max _{\substack{T>T_{0} \\
T \leq N^{5 / 12} / q}}\right\} \\
& \ll_{\epsilon}\left\{q^{3 / 4} T_{0}^{1 / 4} N^{11 / 16}+q^{3 / 4} N^{11 / 16}+N+T_{0}{ }^{-1} N\right\} N^{\epsilon} .
\end{aligned}
$$

Combining (7.12) and (7.13) in (7.8) and then returning to (7.2) and (7.6), we complete the proof of (1.17).

To prove (1.18) we proceed as above except that, in the range $7 / 11 \leq \sigma \leq 3 / 4$, we apply instead the estimate (7.11).

To obtain (1.19) we decompose the integral as

$$
\int_{1 / 2}^{7 / 11}+\int_{7 / 11}^{3 / 4}+\int_{3 / 4}^{4 / 5}+\int_{4 / 5}^{\theta}+\int_{\theta}^{1}
$$

The first three integrals are, as for (1.18), estimated using (7.10), (7.11), and (7.9) respectively. The fourth integral is estimated using (1.7) which holds for $\sigma \geq 4 / 5$ by [HJ]. The fifth integral vanishes by hypothesis. This gives $(1.19) ;(1.20)$ is similar except that we use the density conjecture to treat the interval $[1 / 2+\epsilon, \theta]$. This completes the proof of Theorem 5. 
Lemma 5. For any real $c>0$, we have

$$
\sum_{Q} \frac{1}{\phi^{c}(q)} \int_{\theta}|S-J|^{2}|J|^{2} \ll N^{2 \Theta+1}(\log N)^{4}
$$

and, for $c=0$, the same result holds if $(\log N)^{4}$ is replaced by $(\log N)^{5}$.

Proof. To obtain (7.14) we use an estimate which generalizes a result of Özlük, [Oz, Lemma 11], namely

$$
\sum_{\substack{1 \leq a \leq q \\(a, q)=1}} \int_{-\delta}^{\delta}|S-J|^{2} \ll \delta q N^{2 \Theta}(\log q N)^{4} .
$$

Lemma 5 follows immediately from (7.15) since the left side of (7.14) is

$$
\begin{aligned}
& \ll \sum_{q \leq Q} \frac{\mu^{2}(q)}{\phi^{2+c}(q)} \sum_{\substack{1 \leq a \leq q \\
(a, q)=1}} \int_{-1 / q Q}^{1 / q Q}|S-J|^{2} \min \left(N^{2}, 1 / \beta^{2}\right) d \beta \\
& \ll N^{2} \sum_{q \leq Q} \frac{\mu^{2}(q)}{\phi^{2+c}(q)} \sum_{\substack{1 \leq a \leq q \\
(a, q)=1}} \int_{0}^{1 / N}|S-J|^{2} \\
& +\sum_{j=1}^{\infty} \frac{N^{2}}{2^{2 j}} \sum_{q \leq Q} \frac{\mu^{2}(q)}{\phi^{2+c}(q)} \sum_{\substack{1 \leq a \leq q \\
(a, q)=1}} \int_{2^{j-1} / N}^{2^{j} / N}|S-J|^{2},
\end{aligned}
$$

giving the result.

To prove (7.15) we follow the argument in $[\mathrm{Oz}]$. First note that we may here, as in the proof of Theorem 5 , replace $S_{1}=S-J$ by $S_{2}(\beta ; q, a)$ with an acceptable error, in this case $O(\delta q N)$. Thus we need to prove

$$
\sum_{\substack{1 \leq a \leq q \\(a, q)=1}} \int_{-\delta}^{\delta}\left|S_{2}(\beta ; q, a)\right|^{2} \ll \delta q N^{2 \Theta}(\log q N)^{4} .
$$

By orthogonality of characters,

$$
\begin{aligned}
\sum_{\substack{1 \leq a \leq q \\
(a, q)=1}}\left|S_{2}(\beta ; q, a)\right|^{2} & =\frac{1}{\phi^{2}(q)} \sum_{\substack{1 \leq a \leq q \\
(a, q)=1}}\left|\sum_{\chi(q)} \chi(a) \tau(\bar{\chi}) \Psi(N, \chi, \beta)\right|^{2} \\
& =\frac{1}{\phi(q)} \sum_{\chi(q)}|\tau(\bar{\chi})|^{2}|\Psi(N, \chi, \beta)|^{2} \\
& \ll \frac{q}{\phi(q)} \sum_{\chi(q)}|\Psi(N, \chi, \beta)|^{2} .
\end{aligned}
$$

Thus (7.16) will follow from the estimate

$$
\int_{-\delta}^{\delta}|\Psi(N, \chi, \beta)|^{2} d \beta \ll \delta N^{2 \Theta}(\log q N)^{4} .
$$

Now (7.1) together with the estimate

$$
\psi^{\prime}(x, \chi) \ll x^{\Theta}(\log q x)^{2}
$$


implies on integrating by parts

$$
\Psi(N, \chi, \beta) \ll N^{\Theta}(1+|\beta| N)(\log q N)^{2} .
$$

This estimate immediately proves (7.17) in the case $\delta \leq 1 / N$. We may therefore assume henceforth that $\delta>1 / N$. In this case we turn to (7.2). The term $O\left((\log q N)^{2}\right)$ makes a contribution of $O\left(\delta(\log q N)^{4}\right)$ which is acceptable, and it remains to estimate

$$
\int_{-\delta}^{\delta}\left|\sum_{|\gamma| \leq N^{2}} \mathcal{I}_{\rho}\right|^{2} d \beta .
$$

For zeros in the range $5 \pi \delta N<|\gamma| \leq N^{2}$ we use the van der Corput estimate (7.5) which gives $\mathcal{I}_{\rho} \ll N^{b}|\gamma|^{-1}$ and hence these zeros make a contribution to (7.18) of

$$
\ll \delta N^{2 \Theta}(\log q N)^{4} .
$$

Finally, using Plancherel's theorem,

$$
\int_{-\delta}^{\delta}\left|\sum_{|\gamma| \leq 5 \pi \delta N} \mathcal{I}_{\rho}\right|^{2} d \beta \leq \int_{-\infty}^{\infty}\left|\sum_{|\gamma| \leq 5 \pi \delta N} \mathcal{I}_{\rho}\right|^{2} d \beta=\int_{2}^{N}\left|\sum_{|\gamma| \leq 5 \pi \delta N} u^{\rho-1}\right|^{2} d u
$$

This last expression is

$$
\sum_{|\gamma|,\left|\gamma^{\prime}\right| \leq 5 \pi \delta N} \int_{2}^{N} u^{\rho+\overline{\rho^{\prime}}-2} d u
$$

We claim

$$
\int_{2}^{N} u^{\rho+\overline{\rho^{\prime}}-2} d u \ll \frac{N^{2 \Theta-1} \log N}{1+\left|\gamma-\gamma^{\prime}\right|} .
$$

To see this, note first the left hand side of (7.20) is in absolute value

$$
\leq \int_{2}^{N} u^{b+b^{\prime}-2} d u
$$

which, if $b+b^{\prime} \leq 1+1 / \log N$, is

$$
\leq \int_{2}^{N} u^{-1+1 / \log N} d u \ll N^{1 / \log N} \log N \ll \log N,
$$

while, if $b+b^{\prime}>1+1 / \log N$, is

$$
\leq \frac{N^{b+b^{\prime}-1}}{b+b^{\prime}-1} \ll N^{2 \Theta-1} \log N .
$$

Since $\Theta \geq 1 / 2$, this last estimate covers both cases, and this proves (7.20) for $\left|\gamma-\gamma^{\prime}\right| \leq 1$. On the other hand, if $\left|\gamma-\gamma^{\prime}\right|>1$, the left hand side of (7.20) is

$$
=\frac{N^{b+b^{\prime}-1+i\left(\gamma-\gamma^{\prime}\right)}+O(1)}{b+b^{\prime}-1+i\left(\gamma-\gamma^{\prime}\right)} \ll \frac{N^{2 \Theta-1}}{\left|\gamma-\gamma^{\prime}\right|},
$$

which proves (7.20) in this case. 
Now using (7.20) we see the expression in (7.19) is

$$
\begin{aligned}
& \ll N^{2 \Theta-1} \log N \sum_{|\gamma| \leq 5 \pi \delta N} \sum_{\left|\gamma^{\prime}\right| \leq 5 \pi \delta N} \frac{1}{1+\left|\gamma-\gamma^{\prime}\right|} \\
& \ll N^{2 \Theta-1} \log N \sum_{|\gamma| \leq 5 \pi \delta N}(\log |\gamma| q N)^{2} \\
& \ll \delta N^{2 \Theta}(\log q N)^{4},
\end{aligned}
$$

which completes the proof of (7.17).

\section{Proof of Theorem 1}

We argue as in Section 4 but, as we are not concerned about the loss of logarithmic factors, it is possible to be more brief. Start with the case $k=3$. Here

$$
\begin{aligned}
\sum_{Q} \int_{\theta}\left(S^{3}-J^{3}\right) & \ll \sum_{Q} \int_{\theta}|S-J||J|^{2}+\sum_{Q} \int_{\theta}|S-J|^{2}|J|+\sum_{Q} \int_{\theta}|S-J|^{3} \\
& =E_{1}+E_{2}+E_{3}
\end{aligned}
$$

By $(2.2)$,

$$
E_{1} \ll \sup _{\alpha}|S-J| \sum_{Q} \int_{\theta}|J|^{2} \ll S^{*}(Q) N \log N
$$

and by (2.3),

$$
E_{3} \ll \sup _{\alpha}|S-J| \sum_{Q} \int_{\theta}|S-J|^{2} \ll S^{*}(Q) N \log N .
$$

We estimate $E_{2}$ differently than before. By the Cauchy-Schwarz inequality, by (8.2), and (8.3), we have

$$
\begin{aligned}
E_{2} & \leq\left(\sum_{Q} \int_{\theta}|S-J||J|^{2}\right)^{1 / 2}\left(\sum_{Q} \int_{\theta}|S-J|^{3}\right)^{1 / 2} \\
& \ll S^{*}(Q) N \log N .
\end{aligned}
$$

Using the fact that $q T_{0} \leq Q+Q^{-1} N$ for $|\beta| \leq 1 / q Q$, we deduce from (1.19) that

$$
\begin{gathered}
S^{*}(Q) \ll\left\{Q^{1 / 2} N^{1 / 2}+Q^{-1 / 2} N+Q^{7 / 22} N^{7 / 11}+Q^{-7 / 22} N^{21 / 22}+Q^{1 / 10} N^{3 / 4}\right. \\
\left.+Q^{-1 / 10} N^{17 / 20}+N^{4 / 5}+N^{\Theta}\right\} N^{\epsilon},
\end{gathered}
$$

and so, for $Q=N^{1 / 2} / \log N$, we have

$$
\mathcal{S}_{*} \ll N^{\Theta+\epsilon}+N^{4 / 5+\epsilon} .
$$

Thus by (8.1)-(8.5)

$$
\sum_{Q} \int_{\theta}\left(S^{3}-J^{3}\right) \ll N^{1+\Theta+\epsilon}+N^{9 / 5+\epsilon}
$$

From (3.3) we recall that

$$
\sum_{Q} e\left(\frac{-N a}{q}\right) \int_{\theta} J^{3}(\beta) e(-N \beta) d \beta=\frac{1}{2} N^{2} \mathfrak{S}_{3}(N)+o\left(N^{3 / 2}\right) .
$$


This, combined with (8.6), completes the proof of Theorem 1(a) in the case $k=3$, the proof of Theorem 1(b) being the same except that (1.20) is used in place of (1.19).

Now let $k \geq 4$. We replace (8.1) by an expansion as the sum of $k$ sums and reduce the intermediate sums to the two extremal ones as in (8.4) but using, in place of Cauchy-Schwarz, Hölder's inequality in the form

$$
\sum_{Q} \int_{\theta}|S-J|^{m}|J|^{k-m} \leq\left(\sum_{Q} \int_{\theta}|S-J||J|^{k-1}\right)^{\frac{k-m}{k-1}}\left(\sum_{Q} \int_{\theta}|S-J|^{k}\right)^{\frac{m-1}{k-1}} .
$$

Thus we have, in the notation of (3.2),

$$
\sum_{Q} \int_{\theta}\left(S^{k}-J^{k}\right) \ll E_{1}(k)+E_{k}(k) .
$$

By the same argument used to obtain (2.14), but with Lemma 5 in place of Lemma 2 , we obtain

$$
E_{1}(k)=\sum_{Q} \int_{\theta}|S-J||J|^{k-1} \ll N^{k-2+\Theta}(\log N)^{5 / 2} .
$$

By (2.9) we have

$$
E_{k}(k) \ll \sup _{\alpha}|S-J|^{k-2} \sum_{Q} \int_{\theta}|S-J|^{2} \ll\left(S^{*}(Q)\right)^{k-2} N \log N,
$$

and by (8.5) we see this is

$$
\ll N^{(k-2) \max \{\Theta, 4 / 5\}+1+\epsilon} .
$$

When $k \geq 5$ this is $o\left(N^{k-2+\Theta}\right)$, while for $k=4$ this is $o\left(N^{k-2+\Theta}\right)$ when $\Theta>3 / 5$. On the density hypothesis this condition can be dropped, since the $4 / 5$ may be replaced by $3 / 4$ in the above estimate. This completes the proof of Theorem 1 .

\section{REFERENCES}

[BH] R. C. Baker and G. Harman, Diophantine approximation by prime numbers, J. London Math. Soc. 25 (1982), 201-215. MR 84g:10067

[Da] H. Davenport, Multiplicative Number Theory, 2nd ed., revised by H. L. Montgomery, Springer-Verlag (Berlin), 1980. MR 82m:10001

[Go] D. A. Goldston, On Hardy and Littlewood's contribution to the Goldbach conjecture, Proc. Amalfi Conf. on Analytic Number Theory (Sept. 1989), Univ. Salerno, 1992. MR 94m:11122.

[HL] G. H. Hardy and J. E. Littlewood, Some problems of 'Partitio Numerorum': III On the expression of a number as a sum of primes, Acta Math. 44 (1923), 1-70.

[H-B] D. R. Heath-Brown, Zero density estimates for the Riemann zeta-function and Dirichlet L-functions, J. London Math. Soc. 19 (1979), 221-232. MR 80i:10055

[Hu] M. N. Huxley, Large values of Dirichlet polynomials, III, Acta Arith. 26 (1975), 435-444. MR 52:10620

[HJ] M. N. Huxley and M. Jutila, Large values of Dirichlet Polynomials, IV, Acta Arith. 32 (1977), 297-312. MR 58:5550

[In] A. E. Ingham, Some asymptotic formulae in the theory of numbers, J. London Math. Soc. 2 (1927), 202-208.

[Li] Yu. V. Linnik, A new proof of the Goldbach-Vinogradov theorem, Mat. Sb. 61 (1946), 3-8.

[Mo] H. L. Montgomery, Topics in Multiplicative Number Theory, Lecture Notes in Mathematics, vol. 227, Springer-Verlag (Berlin), 1971. MR 49:2616 
[MV] H. L. Montgomery and R. C. Vaughan, Error terms in additive prime number theory, Quart. J. Math. Oxford (2) 24 (1973), 207-216. MR 49:2624

[Oz] A. E. Özlük, Pair correlation of zeros of Dirichlet L-functions Thesis, University of Michigan, 1982.

[Ti] E. C. Titchmarsh, The Theory of the Riemann Zeta-Function, 2nd ed. revised by D. R. Heath-Brown, Clarendon (Oxford), 1986. MR 88c:11049

[Va1] R. C. Vaughan, Sommes trigonométriques sur les nombres premiers, C. R. Acad. Sci. Paris Ser. A 258 (1977), 981-983. MR 58:16555

[Va 2 ] R. C. Vaughan, The Hardy-Littlewood Method, Cambridge Tracts in Math., vol. 80, (Cambridge), 1981. MR 84b:10002

[Vi] I. M. Vinogradov, Representation of an odd number as a sum of three primes, Dokl. Akad. Nauk SSSR 15 (1937), 6-7.

[Yi] C. Y. Yildirim, The pair correlation of zeros of Dirichlet L-functions and primes in arithmetic progressions, Manuscripta Math., vol. 72, 1991, pp. 325-334. MR 93b:11111

Department of Mathematics, University of Toronto, Toronto, Ontario M5S 3G3, CANADA

E-mail address: frdlndr@math.toronto.edu

Department of Mathematics and Computer Science, San Jose State University, San Jose, California 95192

E-mail address: goldston@sjsumcs.sjsu.edu 